\title{
LA « BIBLIOTHÈQUE DE TRAVAIL » DE ROGER JOSEPH BOSCOVICH. LES SOURCES DU JOURNAL D'UN VOYAGE DE CONSTANTINOPLE EN POLOGNE (1772)
}

Massimo Scandola, Université de Tours, Tours, massimo.scandola@univ-tours.fr

10.31902/fll.29.2019.7

UDK 821.133.1-922.09"17“

UDK 821.163.4-922.09"17“

Résumé: Cet essai analyse les sources consultées par Roger Boscovich dans la rédaction du Journal d'un voyage de Costantinople en Pologne (1772). Il retrace sa « bibliothèque de travail » essayant de comprendre quelques dynamiques de transferts culturels des Lumières à son récit de voyage. J'étudie d'abord le contexte de rédaction, ensuite je propose l'hypothèse de réécriture du récit de voyage à partir de l'étude de la littérature géographique et historique de son époque sur les principautés tributaires de l'Empire ottoman.

Mots-clés : transferts culturels, Balkans, études française, études italiennes, littérature de voyages, Dix-huitième siècle, Lumières.

\section{Introduction}

Dans sa Bibliographie moderne de France (1807), Joseph Marie Quérard énumère les traités de Roger Joseph Boscovich (1711-1787), un jésuite et mathématicien de Raguse, italianisant, qui a vécu en Italie et en France dans la seconde moitié du XVIII ${ }^{\mathrm{e}}$ siècle.

Parmi les ouvrages indiqués par Quérard, deux traités retiennent notre attention : les deux éditions du Journal d'un voyage de Constantinople en Pologne, traduit en français par Pierre-Michel Hennin à Lausanne en 1772 (Boscovich a) et paru en italien en 1784 (Boscovich b; Boscovich c).

Selon un procédé pédagogique, l'auteur s'emploie à transmettre à ses lecteurs tout un patrimoine immatériel, notamment linguistique, propre aux régions de la Bulgarie ottomane et de la principauté de Moldavie.

À partir de ce livre, nous tenterons de remonter aux lectures de Boscovich, aux sources utilisées par l'écrivain, en essayant de comprendre quelle était sa " bibliothèque de travail » et comment elle a influencé ses perspectives analytiques ainsi que le contenu même de sa narration.

\section{Le contexte historique et la rédaction du Journal}

Ces dernières années, l'œuvre, la pensée et les réseaux de sociabilité de Roger Joseph Boscovich (1711-1787) ont fait l'objet de quelques recherches, 
trop rares (Surdich ; Stavischi 973-979 ; Tolomeo 243-263). Marco Martin a édité un commentaire à la version italienne du Giornale di viaggio en 2014 (Martin 171-218; Martin 171-178). Par ailleurs, un projet d'édition de l'œuvre complète de Boscovich est en cours depuis 2011 sous la direction de l'Académie Nationale des Sciences dite des XL, de l'Académie des Sciences et des Arts de la Croatie, de l'Observatoire de Brera et de l'Université Grégorienne de Rome (Edizione nazionale). Toutefois, ses œuvres restent très peu connues et sa correspondance est très peu étudiée, bien que, tout de suite après sa mort, des éloges funèbres aient été publiés et aient circulé dans les bibliothèques scientifiques (Fabroni VII-XLVI ; Bajamonti ; Lalande 411-418). Comme le mentionne la notice du Dizionario biografico degli Italiani, Boscovich était un philosophe adhérant au newtonisme et à la pensée de Leibniz, ses recherches concernaient la philosophie naturelle, l'astronomie, la physique et l'optique (Casini 221-230).

Parmi ses 44 traités scientifiques, presque tous écrits en latin, on peut compter un récit de voyage, le Journal d'un voyage de Constantinople en Pologne (1772), qui est un texte de nature mixte, portant à la fois les caractéristiques de l'essai politique et du récit de voyage.

\section{Le Journal : un cas de réécriture du voyage?}

Le voyage de Constantinople à Camainec se déroule du 24 mai 1762 jusqu'au 15 juillet de la même année, pendant la guerre de sept ans (17561763). Boscovich accompagne l'ambassadeur britannique, Mr. James Porter, ses deux enfants, sa femme qui était la fille d'Albert d'Hochepied, l'ambassadeur des Pays-Bas à Constantinople, le docteur McKenztie, médecin de l'ambassadeur et de sa famille, le beau-frère de l'ambassadeur, le baron Gerard d'Hochepied et Charles Hubsch le secrétaire de l'ambassadeur du roi de Pologne (Boscovich a 1-2 ; Boscovich b IX-X). Enfin, Hadji Abdulla Vizir Aga, officier turc du grand Visir (le Michmandar) gérait tous les besoins des voyageurs. II s'occupait de changer les voitures, de régler tous les frais de déplacement et il était aidé par une garnison de janissaires qui devaient protéger les diligences dans les petits villages (5). Dans son Journal, Boscovich recense deux grandes voitures à cheval et une diligence plus petite ; il comptait également des charrettes avec les lits, les draps, la lingerie, la nourriture et tous les nécessaires pour la cuisine (7).

Avant de réfléchir sur les traités de Boscovich, il faut tenir compte de deux chronologies : l'une biographique et l'autre éditoriale.

La traduction française du Giornale di viaggio est réalisée à partir du manuscrit de Boscovich et elle est publiée en 1772 dix années après le voyage.

Il faudra attendre encore douze années pour que l'édition italienne paraisse chez les typographes Remondini à Bassano, dans la République de Venise (1784). Cette édition est enrichie d'une dédicace à Charles Gravier de Vergennes, secrétaire des Affaires étrangères de Louis XVI et ambassadeur à 
Constantinople à l'époque du voyage. En outre, l'édition italienne est enrichie d'une longue introduction, dans laquelle Boscovich explique à son lecteur les démarches qui ont guidé son analyse. L'auteur a choisi trois verbes clefs ( « observer ", " examiner " et "réfléchir ") afin de décrire l'état politique, civil et social de la Bulgarie ottomane, de la principauté de Moldavie et, enfin, de la Pologne (X). De plus, l'édition italienne de Boscovich est complétée par la Relation des ruines de Troie, c'est-à-dire la description d'un voyage qu'il a fait avec Pietro Correr, qui était le bailo à Constantinople (c'est-à-dire l'ambassadeur et le chef de la communauté vénitienne à Constantinople), avant d'arriver dans la capitale de l'Empire ottoman en septembre 1761 (Martin 38, 96, 143 ; Martin 175-183).

Dans son analyse sur les récits de voyage en Pologne, Michel Marty distingue trois degrés d'analyse d'un récit de voyage (66). Le premier est informatif, et il est consacré à la description les itinéraires et les protagonistes du voyage. Le second est narratif : il transmet les renseignements sur la géographie et sur les institutions. Le troisième degré est psychologique : il présente les expériences vécues par le narrateur pendant son périple avec des stratégies propres aux égo-documents. À ces trois niveaux d'analyse, il convient d'ajouter le quatrième degré d'analyse qui est propre aux " essais " (Marty 94) et qui tente de distinguer les éléments fictionnels des sources d'information. Grâce à cette dernière instance, Michel Marty propose un bilan du voyage qui est complété par les renseignements tirés du « recours à la bibliothèque » (96).

À partir de l'analyse attentive des deux textes (français et italien), nous pouvons affirmer que Boscovich a réécrit le récit du voyage à partir des données tirées des traités de géographie et d'histoire politique de son époque sur l'Empire ottoman et les principautés de cette région. Ces repères sont la « bibliothèque idéale " à laquelle Boscovich fait recours pour rédiger son essai.

De même, la dimension narrative qui a été toujours évoquée par les études précédentes joue un rôle prépondérant, notamment dans l'introduction de la version italienne. À ce propos, Boscovich explique les différents procédés de l'écriture du voyage. L'auteur transmet qu'il a noté, pendant le voyage en carrosse, tous les " pays très peu connus ", tous les "lieux ", tous les horaires d'arrivées (XV). De plus, il souligne qu'il n'avait pas avec lui des « instruments fondamentaux " pour identifier les lieux et la topographie (XVI). En outre, il admet que le manque de connaissance de la langue ne lui a pas permis « d'observer des objets " (XVII). Toutefois, il donne aux lecteurs des repères importants sur l'origine des mots, sur l'étymologie et sur la toponymie. À la fin de son introduction seulement, il avoue que le but de son essai est de décrire le " gouvernement ", " les productions " et " le commerce " de la Moldavie, un " pays très peu connu ", qui était le théâtre de beaucoup de guerres (XVII). L'essai est caché sous la narration. Néanmoins, la dimension narrative renforce le récit du Journal (1772) et du Giornale (1784). L'ensemble s'organise selon la 
chronologie du voyage qui constitue le cadre dans lequel l'abbé Boscovich propose ses réflexions concernant des domaines différents : le système politique et l'organisation administrative, la toponymie, la religion et les langues.

\section{Le système politique et l'organisation administrative}

Avant de commencer sa dissertation, l'abbé Boscovich affirme avoir reçu toutes les données sur les principautés roumaines de Monsieur de la Roche, ministre du prince de Moldavie, et de Monsieur Mille, staroste de Ciarnouz (Boscovich a 252 ; Boscovich b 58 ; Boscovich c 104, Martin 193).

Après une lecture attentive du texte, nous pouvons repérer les sources utilisées par Boscovich. Les formules descriptives du voyage évoquent celles des Voyages de M. Quiclet à Constantinople par terre, enrichis d'annotations par le sieur Promé, marchand libraire (1664), qui était très diffusée en France et en Italie (Quiclet 123-124; Brummet 271).

Sans le reconnaître, Boscovich emprunte à la Descriptio Moldaviae (1711) du prince Demetrius Cantemir (1673-1723) les passages consacrés aux informations relatives à la population et à la topographie (Cantemir b 3-6, 10-23) ; la description des institutions politiques est redevable à l'Histoire de l'Empire ottoman (1713) de Demetrius Cantemir (Cantemir a ; Lemny 23-36). Boscovich n'avoue nulle part ses sources bibliographiques contemporaines. Ce sont ces tendances historiographiques qui peu à peu ont orienté l'opération de réécriture de Boscovich. C'est cette combinaison d'histoire politique et de géographie qui se reflète dans son récit de voyage. Les œuvres de Demetrius Cantemir, qui sont publiées de 1711 jusqu'à 1719 , circulent dans toutes les bibliothèques érudites de France et d'Allemagne (Liu 421-439). Comme le rappelle Stefan Lemny, le traité de Cantemir " a traversé ainsi le $18^{\mathrm{e}}$ siècle, sans perdre de son intérêt " (32). De plus, la presse périodique des années 1750 commence à s'intéresser aux événements politiques de l'Empire ottoman et de ses principautés tributaires. Le Journal universel, ou mémoire pour servir à l'histoire civile avait consacré des comptes rendus aux œuvres de Cantemir. En particulier, un article paru en juillet 1744 résumait l'Histoire de l'Empire ottoman et donnait des renseignements aux lecteurs sur la vie et les œuvres du prince moldave (Journal universel 439-442). Toujours en 1744, le journaliste Henri Du Sauzet consacre une notice biographique à Demetrius Cantemir dans la Bibliothèque française et y propose une liste détaillée de ses œuvres inédites et publiées (Sauzet 213-231; Sauzet 207-208).

Les sujets traités dans le Giornale de Boscovich reflètent l'intérêt de la République littéraire envers l'histoire politique et les institutions des principautés tributaires. Bien que l'abbé ne consacre aucun chapitre à l'histoire, le contexte contemporain reste toujours bien expliqué. Les sultanats d'Achmet III, de Mustafa III (1711-1774) sultan à partir du 1754, d'Abdul Amid I (1774-1789) et Selim III (1761-1808) constituent le cadre dans lequel le récit se développe. 
En outre, Boscovich est très attentif à la machine administrative de l'empire, ce qui se remarque, notamment par le fait qu'il explique les rôles des officiers du vizir. Par exemple, la description de l'office du michmandar est très détaillée : " c'est le nom de l'officier turc que la Porte accorde aux Ministres étrangers pour les accompagner avec un commandement par lequel il est ordonné que dans tout ce qu'ils traversent des États du Grand Seigneur on ait à leur fournir tout ce qui sera nécessaire pour leurs voyages, comme vivres, voitures, chevaux " (Boscovich a 5 ; Boscovich c 6 ; Boscovich b 15). Les michmandars " ont coutume de faire un commerce de ces articles [argent, chariots, provisions], sur lesquels ils gagnent gros ॥ (Boscovich a, 32 ; Boscovich b 24 ; Boscovich c 15 ; Martin 115 ; Martin 174 ; Martin, 178, 189, 191, 199). Ils louent des chariots et ils gardent le commandement, c'est-à-dire le document qui désigne l'autorité du michmandar.

Boscovich offre à son lecteur de nombreuses informations sur les principautés tributaires de l'Empire ottoman. II affirme également avoir reçu ces informations de Monsieur de la Roche qui avait consulté une histoire manuscrite de la Moldavie rédigée par le prince moldave Gregoire Sikka (Gregoiro Ghica). Il est possible qu'il ait complété les informations reçues avec d'autres tirées des manuels français introductifs à l'histoire à la mode. Quoi qu'il en soit, il a remanié le texte final de la version française, puis de l'italienne. Par exemple, la plupart des renseignements concernant les principautés roumaines résument la notice consacrée à la "Moldavie " du Grand dictionnaire historique de Louis Moréri (1759) complété par des informations tirées de deux notices de l'Histoire de Cantemir. L'article du Grand dictionnaire de Moréri renseigne Boscovich sur la chronologie générale et sur l'histoire politique du $16^{\mathrm{e}}$ siècle (Moréri 317-318). En revanche, la première notice du manuel de Cantemir, qui s'intitule "Des deux Moldavies », renseigne sur la capitale Jessi. Elle décrit les frontières géographiques de la Moldavie et renvoie aux sources latines (par exemple, l'épisode d'Ovide revient dans le texte de Boscovich qui n'indique pas sa source) (Boscovich a 200 ; Boscovich b 85 ; Boscovich c 48 ; Cantemir 222 ; Martin 193). En ce qui concerne l'emprunt de la seconde notice, qui est plutôt consacrée à la " création des princes de Moldavie ", Boscovich réécrit le récit du sacre du prince de Moldavie auquel il avoue avoir participé (Cantemir 223). Les rites grecs de sacre des princes se déroulent en présence du vizir ottoman et du patriarche de Constantinople (Cantemir 223). À l'occasion de cette cérémonie religieuse et politique, Boscovich explique brièvement les institutions moldaves. II faut rappeler également que les érudits et les lecteurs de la seconde moitié du $18^{\mathrm{e}}$ siècle accordent de l'importance à ces rites ; Jean-Louis Carra consacre, par exemple, un chapitre de son œuvre, L'Histoire de Moldavie et Valachie paru en 1777, à la cérémonie du sacre du prince de Moldavie (17-21). 
Après son sacre, le nouveau prince Gregorius Calimachi reçoit dans sa cour, dite la Formosa, la délégation diplomatique de l'ambassadeur anglais accompagné par Boscovich. Boscovich explique à son lecteur que le pouvoir du prince moldave repose sur l'appui des Bojari ; en fait, le prince " est obligé d'avoir beaucoup d'égard pour eux, parce qu'il y a beaucoup de princes déposés sur les plaintes qu'ils avoient faites contre eux à la Porte " (Boscovich a 246 ; Boscovich b 101 ; Boscovich c 57). À cette époque, les principautés roumaines sont intégrées dans le système politique et administratif de l'Empire ottoman, la Porte renforce son contrôle de la production de céréales et des matières premières et les princes sont élus parmi la noblesse grecque du Phanar dépendante de Constantinople (Bulguru 73-78). Pour sa part, Boscovich choisit de transmettre ces informations en disant que "le Prince demeuroit peu de temps en place et dépensoit beaucoup pour obtenir cette dignité, lui le premier, et ensuite tous les Grecs qui occupent quelque emploi, cherchent par toutes sortes de voyes à se procurer de l'argent, volant, extorquant, et dépouillant tous ceux qu'ils peuvent " (Boscovich a 216 ; Boscovich 91 ; Boscovich c 51 ; Martin 194-195). L'auteur met l'accent sur le système de gestion et de transmission du pouvoir et relève que " ce prince se choisit entre les Grecs, sujets de la Porte, qui d'une condition presque servile dans laquelle ils gémissent à Constantinople passent en ce pays à des charges de grande autorité et forts lucratives » (Martin 194-195).

Dans son récit, Boscovich signale aux lecteurs que les Boyards (" députés » dans la traduction française du traité de Demetrius Cantemir) (222) appartiennent à l'aristocratie orthodoxe des principautés tributaires. L'auteur explique que " cette noblesse est composée de trois différents grades ; celle du premier s'appelle Bojari, ce nom tire certainement son étymologie du mot slave Boi, qui ainsi que je l'ai déjà dit, signifie guerre, les armes ayant comme ailleurs donné le premier rang à la noblesse. [...] Il y a à la cour du prince douze grandes charges dont il[s] dispose[nt] » (Boscovich a 244 ; Boscovich b 99 ; Boscovich c 56). Pour décrire cet office, Boscovich a utilisé des sources différentes complétées par les articles consacrés aux voïvodes dont il retient l'étymologie du mot bojar. Évidemment, les traités et les récits de voyage du $17^{\mathrm{e}}$ siècle donnaient très peu d'information à ce sujet. Dans la Cosmographie moscovite d'André Thevet (1575), un livre encore très à la mode au $18^{\mathrm{e}}$ siècle, le mot boyard apparaît une seule fois (" un gentilhomme, qu'ils nomment boyard, vault en témoignage, plus que beaucoup d'autres ») (155). De plus, la notice consacrée à ce mot du Glossarium mediae et infimae latinitatis est très laconique (689c). Pour combler ce vide, Boscovich a tiré quelques informations des dictionnaires de son époque. C'est le cas de l'article du Dictionnaire de Trévoux expliquant que « boyard est aussi le nom qu'on donne aux nobles de Transylvanie qui sont parens et alliés des anciens vaivodes. Ce nom signifie seigneur », dont la notice est écrite à l'emprunt de l’article du Grand dictionnaire de Moréri (20). 
Lorsque Boscovich mentionne l'office du voïvode, il explique que ce " mot esclavon signifie proprement la même chose que dux belli en latin ; parce que Voi ou Boi signifie guerre, et Vodit conduire ; mais il se prend en plusieurs endroits où la langue dérive de l'esclavon pour gouverneur et en Pologne les palatins s'appellent Voivoda " (Boscovich a 165 ; Boscovich b 73; Boscovich c 41 ; Martin 203). Cette courte explication insérée dans le texte résume la notice "Vayvode " de Demetrius Cantemir dans son Histoire ottomane (1711) (226). De plus, ce texte évoque l'article "VAYVODES, ou WOYWODES » de l'Encyclopédie de D’Alambert et de Diderot : " c'est le nom qu'on donne en langue esclavonne aux gouverneurs des provinces de Valachie \& de Moldavie. Woyna dans cette langue signifie guerre, $\&$ woda, conducteur, dux bellicus. Les Polonois désignent aussi sous le nom de woywodes ou vayvodes, les gouverneurs des provinces appelés plus communément palatins » (865). La lecture attentive de l'article "Vayvode " du Grand dictionnaire historique ou le mélange curieux de I'histoire sacrée et profane propose également une notice que rappelle le texte de Boscovich : "Le duc ou Gouverneur particulier des villes sous un bassa dans l'empire turc. C'est pourquoi les princes de Transylvanie, Moldavie et Valachie aiment mieux le titre de despote, qui signifie seigneur, que celui de vayvode. Voyez Palatin de Pologne. Ricaut, L’Empire ottoman » (Moréri 881).

Ensuite, l'abbé Boscovich décrit l'office du staroste, le gouverneur d'une province de la principauté de Moldavie : « Nous rencontrâmes le gouverneur qui s'appelle staroste (c'est l'unique starostie de Moldavie) ; elle appartenoit autrefois à la Pologne, pays où les gouvernements s'appellent starostie et elle en a gardé le nom qui vient de la langue Esclavonne, dans laquelle staroste signifie la vieillesse ; ainsi, suivant le véritable sens de ce mot, la dignité de staroste répondroit assez à celle du sénateur romain ॥ (Boscovich a 239; Boscovich b 121; Boscovich c 98). Cette dernière périphrase décrivant les « vieux officiers qui ont servi longue temps » est le résumé du chapitre XXII (Des biens des polonois) consacré aux starostes polonais de Relation historique de la Pologne, contenant le pouvoir de ses rois (1687) de Gaspar de Tende. Cet érudit explique à son lecteur que " le mot polonois starosc signifie vieillesse; et que celuy qui possède un de ces biens s'appelle starosta, qui vient du stary qui signifie vieillard » (273; Dessberg 3-17).

Quand Boscovich relate la " guerre cruelle » entre phanariotes pour faire révoquer le nouveau prince, les " extorsions incroyables " (Boscovich a 239 ; Boscovich b 98 ; Boscovich c 73), la corruption, la vénalité des offices de l'administration princière et de l'église, notamment la nomination des évêques et des prêtres, il offre un portrait sombre de la Moldavie. Cet avis est partagé par JeanLouis Carra que dans son Histoire il enregistre que " ce qu'il a de singulier chez ces des potes de Moldavie, c'est que toutes leurs richesses [...] sont toujours dans des malles ou coffres, car ils ont sans cesse à craindre d'être déposés par 
force ou enlevés ou assassinés ; et par cette précaution leur famille peut au moins sauver leurs effets les plus précieux » (106).

\section{La topographie et la toponymie}

Les guerres du $18^{\mathrm{e}}$ siècle avaient modifié les frontières et la géographie politique des principautés de Valachie et Moldavie. La guerre entre la Russie et l'Empire ottoman conduit les savants à s'intéresser à la géographie et à la topographie de ces régions dans les gazettes. La frontière de la Moldavie fait l'objet d'un article (La seconde lettre de Mr. le Major Humbert qui contient quelques remarques de géographie), paru dans la gazette Bibliothèque germanique (1740), dans lequel le rédacteur discute sur "la position de ces deux pays » dans les cartes géographiques « que Sanson, Jaillot et de l'Isle nous ont donné de la Hongrie " (Bibliothèque germanique 36). En vérité, c'est de manière circonspecte mais résolue que ces érudits justifient l'intérêt pour les principautés tributaires de l'Empire ottoman par son lien avec la politique internationale. Boscovich accorde ainsi de l'importance à la géographie politique, à la topographie et au patrimoine toponymique de la Bulgarie ottomane et de la Moldavie. Comme Marco Martin l'indique dans sa dernière étude, Boscovich expose l'origine du toponyme d'une dizaine de villages parmi la centaine qu'il traverse pendant le voyage de Constantinople à Cameniec en Pologne (Martin 187-189). Wendy Bracell mentionne une attitude " ethnographique " dans Boscovich ; c'est-àdire que l'auteur explique s'il s'agit d'un village turc ou d'un village à majorité grecque ; il énumère les églises, les mosquées, les synagogues, les bâtiments publics et les marchés. En particulier, Karaclikioi est un village grec, Kinicly est un petit village avec 24 maisons turques et 15 maisons grecques, Caristran « est un bourg habité par environ cent familles turques " (Boscovich a 37), Kirklisé " c'est une assez grande ville dont les habitants sont pour la plupart turcs, il y a cependant un bon nombre de Grecs : on y voit plusieurs mosquées, une belle fontaine, un beau bain et un bezestein ou marché qui nous parut très pauvre " (Boscovich a 49-50). À l'occasion du dîner chez Monsieur Folley, le trésorier de l'ambassadeur anglais, Boscovich décrit les bezestins, les quartiers des "grandes marchés » de Constantinople et des villes de l'Empire ottoman (Martin 184 ; Dictionnaire de Trévoux 880 ; Savary de Bruslon 937). Boscovich témoigne d'une connaissance approfondie de la toponymie turque : il explique, par exemple, qu' « on compte en Turquie plus de trois cents bourgs ou villages qui portent ce nom [Burgas] parce qu'on l'a donné à tous ceux qui avoient un château fort » (Boscovich a 44; Boscovich c 28 ; Martin 108 ; Martin 189).

Et puis, d'autres lieux publics décrits par Boscovich sont les hans, dont il donne une explication très précise : "Ces Hans ou Kans sont des bâtiments publics, fort grands comme un grand salon, porté sur quatre murailles et couvert d'un large toit. Le toit est d'ordinaire soutenu outre les murailles par un et le plus souvent par deux rangs de colomnes, pilastres ou piliers [...] Chaque Han 
a son gardien et est regardé comme un lieu sacré tant pour les personnes que pour les bagages» (Boscovich a 11 ; Boscovich b 17 ; Boscovich c 8 ; Martin 108 ; Martin 189). Lorsqu'il décrit une ville, un village ou un quartier turc, Boscovich choisit d'utiliser ce mot, hans, comme Claude de La Magdelaine le faisait dans son Miroir ottoman (1677) ( 91 ; Quiclet 62, 64, 127) 1 .

\section{Les religions et les langues}

Le journaliste rédacteur du compte rendu qui paraît dans l'Année littéraire (1773) porte l'attention du lecteur sur ces deux sujets : langue et religion sont deux caractéristiques liées l'une à l'autre dans le Journal de Boscovich ; comme le dit Wendy Bracewell, le regard de Boscovich est " ethnographique " (63), son intérêt pour sa langue natale, l'esclavon qui lui permet de communiquer avec les officiers de la Moldavie, cache peut-être une perspective cosmopolite (Bracewell 61 ; Wolff 172-173).

Dans son Journal, Boscovich ne se limite pas à transmettre des renseignements importants sur la religion des principautés de Moldavie ainsi que sur les langues parlées par les sujets de la Porte, il essaie aussi de proposer à son lecteur une voie d'interprétation. En fait, les historiens, notamment Wendy Bracell, ont déjà souligné la manière selon laquelle Boscovich s'insère dans un contexte littéraire plus ample qui définit les frontières à partir d'un " vocabulaire culturel » déjà codifié par Ricaud et les historiens du $18^{e}$ siècle. À ce propos, Larry Wolff affirme que les érudits du $18^{\mathrm{e}}$ siècle ont créé " l'Europe occidentale et l'Europe orientale ensemble, comme deux principes complémentaires [...] par opposition et par contiguïté " (Wolff 5-6, 173-174; Martin 197). Ainsi, dans cette perspective, Boscovich associe l'Europe à un " espace cultivé " qui s'oppose aux villes de l'Empire ottoman qu'il considère comme des « pays barbares » (Boscovich a 316 ; Boscovich b 125 ; Boscovich c 125).

Toutefois, nous estimons que Boscovich expose une idée plus complexe. Bien qu'il admette ne pas comprendre la langue des boyards de la cour du prince Gregorius Calimachi, l'abbé Boscovich mentionne souvent les liaisons entre la langue parlée en Bulgarie et en Moldavie et l'esclavon. Lorsqu'il arrive dans les villages de la Bulgarie ottomane, il affirme que : « La langue du pays est un dialecte de la langue esclavonne, et comme c'est aussi celle de Raguse, ma patrie, je pus me faire entendre à un certain point et comprendre partie de ce qu'ils disoient " (Boscovich b IX). De même, il admet réussir à s'entendre avec un prêtre orthodoxe : " je pus découvrir pendant le peu temps que je séjournai chez eux, en parlant ma langue, et en les faisant interroger en turc qu'ils ne savent ni le pater ni le credo " (Boscovich a 61 ; Boscovich b 61 ; Martin 207).

1 Le Miroir Ottoman, p. 91 : « II y a des Hans, ou karvansarats, pour la commodité des passants, ces lieux sont de très grande étandüe, faites en façon de grandes hales, et tout à l'entour il y a un terrain élevé de quatre pieds, et d'autant de largeur pour le repos des hommes, et les chevaux sont attachés au bas, et les mulets et chameaux dans le vuide que le bâtiment fait au milieu ». 
Poursuivant le voyage dans cette région, il relate la rencontre avec un vieux " prêtre grec » dit "papas " auquel il a rendu visite; " et à l'aide de la langue esclavonne » il lui a parlé et il a " compris qu'il se trouvoit ce prêtre dans cette contrée qui y avoit une église cachée » (Boscovich a 136 ; Boscovich b 62 ; Boscovich c 34 ; Martin 108, 112, 131, 134, 135, 136).

Une fois que l'ambassade est arrivée à la cour du prince de Moldavie, Boscovich explique que " La langue utilisée dans le pays est un mélange de différentes langues; il y a quelque chose d'esclavon et du turc, mais le latin, l'italien y dominent, il s'y rencontre une quantité des mots italiens qui ne sont pas dérivés du latin, et la terminaison de nombre de mots de cette dernière langue est aussi changée à la manière des Italiens ॥ (Boscovich a 253 ; Bosocivh b 103 ; Boscovich c 58). Encore une fois, Boscovich ne cite pas ses sources, la plupart de ces information sur la langue moldave et ses rapports avec l'italien son tirée de la Descriptio Moldaviae, dans laquelle Demetrius Cantemir explique que les érudits " ex italica dialecto eam derivatam esse arbitrantur » grâce à une longue série d'exemples que Cantemir a tiré des grammaires du $17^{\mathrm{e}}$ siècle (148-149).

\section{Conclusions}

L'analyse de ces passages nous conduit à un constat. S'il est vrai que l'auteur marque la frontière culturelle qui sépare l'Europe occidentale des principautés soumises à l'Empire ottoman, nous ne pouvons pas ignorer le fait qu'il énonce également l'existence d'un patrimoine linguistique commun entre, d'une part, la langue des sujets chrétiens de la Porte et, d'autre part, sa langue maternelle parlée à Raguse. Bien qu'il soit audacieux de parler de panslavisme avant la lettre, nous ne pouvons pas ignorer une telle proximité qui se situe à la limite entre l'ouverture cosmopolite du voyageur du $18^{\mathrm{e}}$ siècle et les germes de la mise en valeur d'un patrimoine linguistique, auquel les élites culturelles du $19^{\mathrm{e}}$ siècle seront particulièrement attentives.

\section{Éditions de l'œuvre de Roger Boscovich}

[Boscovich a]: Boscovich, Roger. Journal d'un voyage de Constantinople en Pologne. Lausanne : Grasset, 1772. Print.

[Bosovich b]: Boscovich, Roger. Giornale di un viaggio da Costantinopoli in Polonia dell'abate Ruggiero Giuseppe Boscovich con una sua Relazione delle rovine di Troja, e in fine il Prospetto delle opere nuove matematiche del medesimo autore, contenute in cinque tomi. Bassano: Remondini, 1784. Print.

[Boscovich c]: Boscovich, Roger. Giornale di un viaggio da Costantinopoli in Polonia dell'abate Ruggiero Giuseppe Boscovich, con una relazione delle rovine di Troia ed infine il prospetto delle Opere nuove matematiche conte- 
nute in cinque tomi, éd. par E. Proverbio, Edizione nazionale delle opere e della corrispondenza di Ruggero Giuseppe Boscovich. Vol. 17/2. S. I.: Edit4, 2008. Print.

\section{Références bibliographiques}

Alexandrescu-Dersca Bulguru, Marie-Matilde. « L'approvisionnement d'Istanbul par les principautés roumaines ". Revue des mondes musulmans et de la Méditerranée 66 (1992) : 73-78. Print.

Bajamonti, Giulio. Elogio storico dell'abate Ruggiero Giuseppe Boscovich. Raguse : s. e., 1789. Print.

Bracewell, Wendy. "The Limits of Europe in East European Travel Writing ", in Under Eastern Eyes. A Comparative Introduction to East European Travel Writing on Europe. Ed. Edited by W. Bracewell et A. Drace-Francis Budapest New York: Central European University Press, 2004. Print.

Brummett, Palmira. Mapping the Ottomans. Sovereignty, Territory, and Identity in the Early Modern Mediterranean. New York: Cambridge University Press: 2004. Print.

Cantemir, Demtrius. Descriptio antiqui et hodierni status Moldaviae. Bucarest : Typographia Curti, 1872 : 3-6, 10-23. Print.

Cantemir, Demetrius. Histoire de l'Empire Ottoman, où se voient les causes de son agrandissement et de sa décadence, trad. par $\mathrm{M}$. de Joncquieres. Paris : Barrois, 1743 . Print.

Carra, Jean Louis. Histoire de la Moldavie et de la Valachie. Jassy : Société Typographique des Deux-Ponts, 1777. Print.

Casini, Paolo. "Boscovich, Ruggero Giuseppe ». Dizionario Biografico degli Italiani. Vol. 13. Rome: Treccani, 1971. Print.

Dessberg, Frédéric. « La Pologne vue de France : un aperçu historiographique ». Revue historique des armées 260 (2010) : 3-17. Print.

Dictionnaire universel françois et latin : vulgairement appelé dictionnaire de Trévoux. vol. 1. Paris : Jésuites et imprimeurs de Trévoux, 1771. Print.

Du Fresne du Cange, Charle. «Bojari : Nobiles inferioris ordinis apud Polonos ". Glossarium mediae et infimae latinitatis, vol. 1, Niort : L. Favre. 1883-1887 : 689c. Print.

Du Sauzet, Henri. « Article III ». Bibliothèque française, ou histoire littéraire de la France 29.2 (1744) : 216-231. Print.

Du Sauzet, Henri. "Article X ». Nouvelle bibliothèque ou histoire littéraire des principaux écrits qui se publient 18 (1744) : 207-208. Print.

Edizione Nazionale delle Opere e della Corrispondenza di Ruggiero Giuseppe Boscovich (Accademia Nazionale delle Scienze detta dei XL - Hrvatska akademija znanosti i umjetnosti - INAF Osservatorio Astronomico di Bre- 
ra-Pontificia Università Gregoriana), Vol. XVII/II Opere letterarie Opere in prosa, Giornale di un viaggio da Costantinopoli in Polonia. Ed. E. Proverbio. Nova Milanese: Edit Editoria Elettronica, 2008.

Fabroni, Angelo. « Elogio dell'abate Ruggiero Giuseppe Boscovich ». Memorie di Matematica e Fisica della Società Italiana 4 (1788): vii-xlvi. Print.

Encyclopédie ou Dictionnaire raisonné des sciences, des arts et des métiers. Ed. Diderot et D'Alembert. Vol. 16. Paris : Briasson, David, Le Breton, Durand, 1751.

Humbert, Pierre. "La seconde lettre de Mr. le Major Humbert qui contient quelques remarques de géographie ». Bibliothèque germanique ou histoire littéraire de l'Allemagne. 17 (1740) : 33-40. Print.

Journal universel, ou mémoire pour servir à l'histoire civile 3 (1744) : 439-441. Print.

Lefrançais de Lalande, Joseph-Jérôme. «Elogio del Boscovich ». Journal des Savants 1 (1792) : 411-418. Print.

Lemny, Stefan. " Approches roumaines de I'histoire ottomane ». Dix-huitième Siècle 28 (1996) : 23-36. Print.

Liu, Nicolae. « Dimitrie Cantemir, prince philosophe de Moldavie. Échos européens ». Dix-huitième Siècle 15 (1983) : 421-439. Print.

Magdeleine, Claude de La. Le Miroir Ottoman. Avec un succinct récit de tout ce qui c'est [!] passé de considérable pendant la guerre des Turqs en Pologne, jusqu'en 1676. Bale : Genath, 1677. Print.

Martin Marco. "Bulgaria e Moldavia attraverso il «Giornale di un viaggio da Costantinopoli in Polonia» di Ruggiero Giuseppe Boscovich ». Itineraria. Letteratura di viaggio e conoscenza del mondo dall'Antichità al Rinascimento 2 (2012): 171-218. Print.

Martin Marco. "Ruggero Giuseppe Boscovich and his Giornale di un viaggio da Costantinopoli in Polonia. A travel diary through Eastern Europe with original scientific observations ". Scientific cosmopolitanism and local cultures: religions, ideologies, societies. Proceedings of 5th International Conference of the ESHS. Ed. Gianna Katsiampoura. Athens: National Hellenic Research Foundation/Institute of Historical Research, 2012: 171178. Print.

Moréri, Louis. "Bojares ». Grand dictionnaire ou Le mélange curieux de l'Histoire sacrée et profane, vol. 9, Paris libraires associés [Le Mercier, Desaint \& Saillant, Jean-Thomas Herissant, Boudet, Vincent, Le Prieur], 1759. Print,

Quiclet, [M.]. Voyages à Constantinople par terre. Paris : Pierre Promé, 1664. Print.

Savary Des Bruslon, Jacques. Dictionnaire universel de commerce, vol. 1. Paris : Veuve Estienne et Fils, 1748. Print. 
Stavinschi, Magda. "Roger Boscovich in Romania ». Memorie della Società Astronomica Italiana 61.4 (1990): 973-979. Print.

Surdich, Francesco. "Prefazione ». Commento al Giornale di un viaggio da Costantinopoli in Polonia di Ruggiero Giuseppe Boscovich. Ed. Marco Martin. Roma : Aracne, 2014. Print.

Tende, Gaspar de. Relation historique de la Pologne. Paris : Jacques Villery, 1687. Print.

Thevet, André. Cosmographie moscovite, recueillie et publiée par le prince Augustin Galitzin. Paris: J. Techener, 1858. Print.

Tolomeo, Rita. "Spunti e riflessioni sulla Moldavia dal Giornale di viaggio di Ruggiero Boscovich ». Romània orientale 11 (1999): 243-263. Print.

Wolff, Larry. Inventing Eastern Europe. The Map of Civilisation on the Mind of Enlightenment. Stanford: Stanford University Press, 1994. Print.

THE « WORKING LIBRARY » OF ROGER JOSEPH BOSCOVICH. THE SOURCES OF THE JOURNAL OF TRAVEL FROM CONSTANTINOPLE TO POLAND (1772)

This essay analyses the sources of the Journal of a Voyage of Costantinople in Poland (1772) of Roger Boscovich. In this essay, I study the context of writing, and so I propose the hypothesis of rewriting the story travels from the study of the geographical and historical literature of his time about the vassal and tributary States of the Ottoman Empire.

Key words: cultural transfers, French studies, Italian studies, travel literature, Balkans, Eighteenth century, Enlightenment. 